\title{
Sarcoma indiferenciado primario de aurícula izquierda
}

\author{
Primary undifferentiated sarcoma of left atrium
}

\author{
Yelson A. Picón-Jaimes ${ }^{1 *}$, Leec F. Ortega-Uribe², Javier E. Orozco-Chinome ${ }^{3}$, \\ Jessica S. Molina-Franky y Mabel P. Franky-Rojas ${ }^{5}$ \\ ${ }^{1}$ Servicio de Consulta Externa, Medicien, Santiago, Chile; ${ }^{2}$ Servicio de Cuidados Intensivos, Clínica Chicamocha, Bucaramanga, Colombia; ${ }^{3}$ Servicio \\ de Consulta Externa, Red Salud, Santiago, Chile; ${ }^{4}$ Programa de Medicina, Universidad de Boyacá, Tunja, Colombia; ${ }^{5}$ División de Acreditación, \\ Universidad de Boyacá, Tunja, Colombia
}

\section{Resumen}

El objetivo es ilustrar al lector sobre un tumor infrecuente en la literatura médica y resumir los conocimientos actuales que se tienen sobre esta patología. Caso clínico: Reportamos el caso de un varón en la quinta década de la vida, a quien se realizó ecocardiograma transtorácico por deterioro de la clase funcional. La ultrasonografía evidenció un tumor que ocupaba el 95\% de la aurícula izquierda, con compromiso hemodinámico e hipertensión pulmonar grave. Luego de estudios de extensión, fue llevado a cirugía para extracción de la masa por esternotomía media. La patología reveló sarcoma de alto grado indiferenciado sin compromiso linfovascular.

Palabras clave: Atrios cardiacos. Insuficiencia cardiaca. Neoplasia. Sarcoma.

\begin{abstract}
This text aimed to illustrate the reader about one of the most infrequent tumors in the medical literature and discuss based on current literature what is known to date of this pathology. Case report: We report the case of a male in the fifth decade of life, who underwent transthoracic echocardiography due to impairment of the functional class. Ultrasound showed tumor lesion that occupied $95 \%$ of the left atrium, with hemodynamic compromise and severe pulmonary hypertension. After extension studies, he was taken to surgery with total mass extraction by middle stereotomy. The pathology revealed undifferentiated high-grade sarcoma without lymphovascular involvement.
\end{abstract}

Key words: Heart atria. Heart failure. Neoplasms. Sarcoma.

\section{Introducción}

Los tumores cardiacos primarios son patologías infrecuentes que pueden afectar a todos los grupos etarios y a ambos sexos, pero son más frecuentes entre la tercera y la sexta décadas de la vida, con predominancia en las mujeres con una relación 5:4 respecto a los hombres ${ }^{1-3}$. Tienen una incidencia reportada de aproximadamente el $0.005 \%$ y la mayoría provienen de metástasis pulmonares ${ }^{1}$. De estos, el $70-75 \%$ corresponde a tumores benignos, el $75 \%$ a mixomas y el $25-30 \%$ a tumores malignos ${ }^{2,3}$. Los sarcomas constituyen menos del $1 \%$ de todos los sarcomas de tejidos blandos y alrededor del $13-20 \%$ de los tumores malignos del corazón ${ }^{4}$.

\section{Correspondencia:}

*Yelson A. Picón-Jaimes

Avda. Pocuro, 2260

Providencia 
El tipo de sarcoma más común es el angiosarcoma (37-41\%), seguido del sarcoma indiferenciado o inclasificable (0-24\%), el histiocitoma fibroso maligno (11$24 \%$ ), el leiomiosarcoma (8-9\%), el rabdomiosarcoma (4-7\%), el osteosarcoma (3-9 \%) y el fibrosarcoma $(0,3-5 \%)^{5,6}$.

La ubicación del tumor dentro de las cámaras cardiacas está asociada con los tipos histológicos: los angiosarcomas se localizan en el corazón derecho y los otros tipos de sarcomas en el corazón izquierdo; a menudo infiltran estructuras como el tabique o la válvula mitral, lo cual lleva a sobrecarga de volumen de la aurícula izquierda con congestión retrógrada e hipertensión pulmonar, desencadenando los principales síntomas ${ }^{1,3,4}$.

Estos tumores producen una sintomatología poco específica y, por lo tanto, son difíciles de diagnosticar. Suelen ser asintomáticos en los estadios iniciales hasta que el tamaño es lo suficientemente importante para generar la aparición de signos de falla cardiaca congestiva por efecto de masa, ocasionando disminución del gasto cardiaco, e incluso pueden presentarse con invasión tumoral al pericardio y producir restricción al llenado de las cámaras cardiacas ${ }^{4,5}$.

El ecocardiograma, la tomografía computada (TC) y la resonancia magnética (RM) son ayudas diagnósticas sensibles que hacen posible la sospecha de estos tumores. En la mayoría de los casos, la hipótesis diagnóstica inicial corresponde a mixoma benigno, pero el diagnóstico definitivo se realiza durante el procedimiento quirúrgico, al observar las características de la lesión y la invasividad, y con el análisis histopatológico ${ }^{6}$. Esta patología está asociada a una pobre sobrevida, con un tiempo de 12 meses poscirugía sin nuevos datos de tumor y un tiempo de sobrevida en general de 9 meses $^{7}$.

El objetivo de este caso es ilustrar al lector sobre uno de los tumores más infrecuentes en la literatura médica y comentar lo que se sabe hasta la fecha de esta patología.

\section{Caso clínico}

Varón en la quinta década de la vida sin antecedentes médicos de importancia, que ingresó para atención ambulatoria y realización de ecocardiograma transtorácico por deterioro de la clase funcional, consistente en disnea de moderados a pequeños esfuerzos de 3 meses de evolución. La ultrasonografía evidenció una lesión tumoral que ocupaba el 95\% de la aurícula izquierda, adherida al techo atrial y comprometiendo la llegada de las venas pulmonares izquierdas superior e inferior, con compromiso hemodinámico, hipertensión pulmonar grave con presión pulmonar sistólica de $100 \mathrm{mmHg}$, aplanamiento del tabique interventricular por sobrecarga de presión de las cavidades derechas y fracción de eyección del ventrículo izquierdo (FEVI) del $35 \%$. El paciente fue enviado al departamento de urgencias, donde se documentó una exploración física general inicial con temperatura de $36.5{ }^{\circ} \mathrm{C}$, presión arterial en el brazo izquierdo de 142/85 mmHg, frecuencia cardiaca de 90 latidos por minuto y saturación de oxígeno al aire ambiente del $95 \%$. Durante la exploración segmentaria se identificó ingurgitación yugular, ruidos cardiacos rítmicos, de baja intensidad, con soplo sistólico de predominio en foco mitral de grado III/VI de Levine, y murmullo pulmonar presente con ligeras crepitancias en ambas bases pulmonares. Por alto riesgo de muerte súbita fue hospitalizado en la unidad de cuidados intensivos para monitorización clínica continua y realización de imágenes diagnosticas (Fig. 1).

El paciente fue valorado por el departamento de cirugía cardiovascular, quienes le realizaron estudios de extensión en búsqueda de metástasis, los cuales fueron negativos. Días después fue llevado al pabellón quirúrgico, donde previa anestesia general y colocación de un catéter venoso central y un catéter en la arteria radial izquierda para monitorización, se le realizó esternotomía media y exposición intrapericárdica. Se le administró heparina a dosis de $3 \mathrm{mg} / \mathrm{kg}$, se le hizo canulación arterial y venosa para iniciarle circulación extracorpórea, y descenso de la temperatura a 32 ${ }^{\circ} \mathrm{C}$. Con pinzamiento de la raíz de la aorta, se le infundió solución cardiopléjica sanguínea fría, logrando el cese de la actividad electromecánica del corazón; en igual sentido, se le abordó por las dos aurículas con apertura del tabique interauricular y se le resecó la masa completa según valoración microscópica (Fig. 2); le fue preservada la válvula mitral. Se realizó resección de la orejuela izquierda y cierre con ArtriClip ${ }^{\mathrm{TM}}$. Se elevó la temperatura a $36^{\circ} \mathrm{C}$ y se despinzó la raíz de la aorta permitiendo la reperfusión, la cual fue exitosa y el paciente retornó a un ritmo sinusal, por lo que se suspendió la circulación extracorpórea y se descanuló. Paso seguido, se comprobó la funcionalidad de la válvula mitral con ecocardiograma transesofágico y a continuación se procedió al cierre por planos y se envió al paciente a recuperación sin complicaciones, luego de 4 horas y 30 minutos de procedimiento.

En la unidad de cuidados intensivos cardiacos se le realizó ecocardiograma transtorácico posquirúrgico 


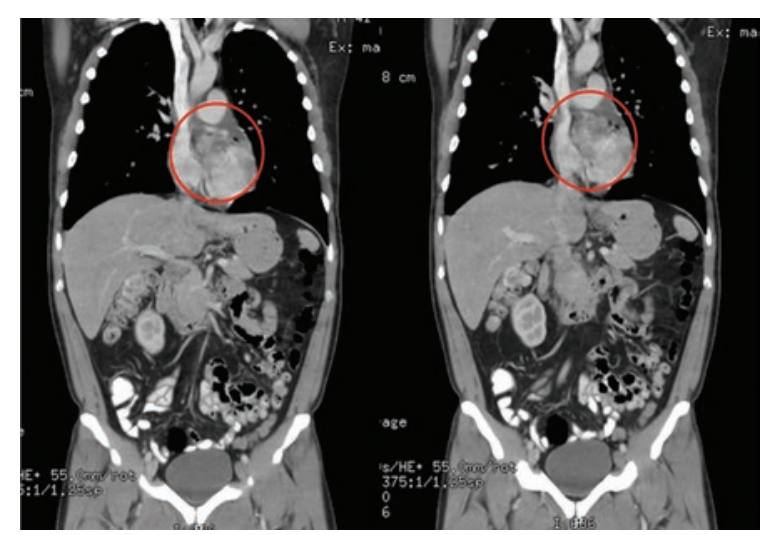

Figura 1. Imágenes diagnosticas en las que se evidencia una lesión tumoral en la aurícula izquierda.

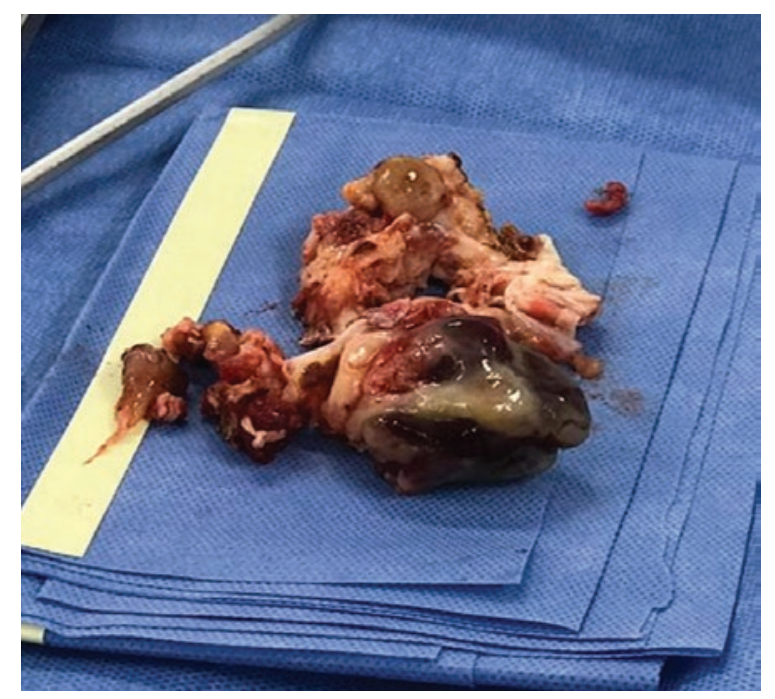

Figura 2. Masa resecada de la aurícula izquierda del paciente.

que reportó ventrículo izquierdo de dimensiones conservadas, hipocinesia leve disíncronica septal, FEVI del $60 \%$, aurícula izquierda sin visualización de material neoplásico en su interior, insuficiencia mitral III/ IV y derrame pericárdico leve sin taponamiento. EI paciente continuó con tendencia a la mejoría y luego de terminar el plan de rehabilitación cardiopulmonar fue dado de alta.

El reporte de patología de la lesión extirpada reveló a la microscopía lesión tumoral constituida por células grandes con marcada atipia citológica, tipo osteoclasto, con hasta tres mitosis por campo; componente inflamatorio constituido por plasmocitos, linfocitos reactivos alrededor de los vasos, con extensas áreas de necrosis tumoral. Índice de proliferación KI 67 del 40\%. Por hallazgos de inmunohistoquímica se determinó lesión compatible con sarcoma de alto grado indiferenciado sin compromiso linfovascular.

\section{Discusión}

El primer reporte de tumor cardiaco se remonta a 1559, por Columbus de Padua, y el primer diagnóstico ante mortem de un sarcoma fue realizado por Barners en $1934^{8}$. Los sarcomas cardiacos primarios son raros, pero representan la mayoría de los tumores cardiacos malignos primarios. Se cuenta con pocos reportes en la literatura, siendo la mayoría de ellos reportes de casos de angiosarcomas ${ }^{5,6,9-11}$.

El principal síntoma que llevo al paciente a consultar al servicio médico fue la disnea; este es uno de los síntomas más frecuentes producidos por la obstrucción del flujo sanguíneo hacia el ventrículo izquierdo, secundario a la obstrucción de una o varias venas pulmonares. Se puede acompañar de insuficiencia cardiaca por ocupación de la masa tumoral o por el fenómeno de enclavamiento tumoral en la válvula mitral, logrando ocasionar síncope o muerte súbita. Adicionalmente, se pueden producir signos asociados a la embolia de las arterias coronarias, los vasos cerebrales o renales, la bifurcación aórtica o las arterias de las extremidades inferiores ${ }^{11,12}$. Sin embargo, la sintomatología de los tumores cardiacos es múltiple e inespecífica, puesto que cualquier síntoma cardiovascular puede ser una manifestación clínica de un tumor cardiaco y, así mismo, una gran parte de los tumores son asintomáticos hasta que por su extensión comprometen el funcionamiento hemodinámico o eléctrico del corazón. Todos estos síntomas con frecuencia llevan a sospechar otras enfermedades cardiovasculares, retrasando el diagnóstico ${ }^{13}$.

Una de las pruebas más sensibles para diagnosticar esta patología es el ecocardiograma transtorácico o transesofágico. Es una prueba fácil de realizar y sensible para detectar lesiones tumorales intracardiacas. Se puede complementar con la TC y la RM torácica y abdominal ${ }^{6,8}$, que muestran la extensión del tumor, la presencia de metástasis, la invasión al mediastino, las infiltraciones al miocardio y la compresión de las cámaras cardiacas. Usualmente, al momento del diagnóstico estas lesiones se encuentran como una neoplasia maligna confinada al corazón, sin evidencia de neoplasia primaria extracardiaca, correspondiendo con la presentación clínica del paciente de nuestro caso ${ }^{2,12}$.

Por otro lado, la radiografía de tórax no se considera una prueba diagnóstica, pero muestra signos sugestivos de patología tumoral cardiaca, como cardiomegalia, infiltrados producto del edema pulmonar secundario a la insuficiencia cardiaca congestiva, derrame pleural, nódulos pulmonares o parálisis del hemidiafragma izquierdo'. 
El tratamiento primario de elección continúa siendo la resección quirúrgica completa, la cual solo es posible en el $33 \%$ de los $\operatorname{casos}^{5}$, y también la resección quirúrgica con autotrasplante, que ha demostrado mejores resultados en los pacientes que presentan tumores de difícil acceso anatómico ${ }^{14-16}$.

La cirugía con márgenes de resección negativos es el único tratamiento exitoso probado hasta ahora, pero la resección tumoral completa solo es posible en menos del $50 \%$ de los pacientes ${ }^{9}$. En este caso, el paciente se abordó mediante resección quirúrgica completa de masa según valoración microscópica, preservando la válvula mitral, con resección de la orejuela izquierda, evitando así la muerte súbita debida a insuficiencia cardiaca.

A pesar de esto, los tratamientos son paliativos y el pronóstico es desfavorable, ya que son tumores altamente agresivos, con una sobrevida a 1 año de identificada la lesión menor del $20 \%{ }^{5}$, debido a la rápida infiltración tumoral, con evidencia de metástasis en el $80 \%$ de los $\operatorname{casos}^{15-17}$. Adicionalmente se observa una alta recurrencia, aun cuando se haya resecado por completo el tumor, por lo cual la terapia adyuvante con quimioterapia y radiación está indicada en todos los pacientes. La terapia multimodal puede lograr una supervivencia razonable para los pacientes con sarcomas cardiacos resecados; incluso los pacientes con recurrencia local o metástasis pueden beneficiarse de un tratamiento agresivo ${ }^{13-18}$.

Lo anterior permite concluir que el diagnóstico de esta patología continúa siendo un reto clínico, debido a su baja incidencia y a la inespecificidad de los síntomas. No se han establecido métodos ideales para su confirmación, pero los estudios de imágenes más utilizados en la actualidad que permiten la identificación del tumor son la RM, la TC con contraste y el ecocardiograma bidimensional, siendo este último el estudio más usado para detectar tumores cardiacos. No obstante, solo el análisis histopatológico puede confirmar y clasificar esta patología².

\section{Agradecimientos}

Al paciente y su familia, quienes amablemente aceptaron las entrevistas posteriores al alta hospitalaria para completar la historia.

\section{Financiamiento}

Recursos propios de los autores.

\section{Conflicto de interés}

Los autores declaran no tener conflictos de intereses.

\section{Responsabilidades éticas}

Protección de personas y animales. Los autores declaran que para esta investigación no se han realizado experimentos en seres humanos ni en animales.

Confidencialidad de los datos. Los autores declaran que han seguido los protocolos de su centro de trabajo sobre la publicación de datos de pacientes.

Derecho a la privacidad y consentimiento informado. Los autores han obtenido el consentimiento informado de los pacientes y/o sujetos referidos en el artículo. Este documento obra en poder del autor de correspondencia.

\section{Bibliografía}

1. Ekmektzoglou KA, Samelis GF, Xanthos T. Heart and tumors: location, metastasis, clinical manifestations, diagnostic approaches and therapeutic considerations. J Cardiovasc Med. 2008;9:769-77.

2. Vilcarromero Arbulú GA. Rabdomiosarcoma cardíaco. Rev Argent Cardiol. 2013;81(2). (Consultado el 2 de octubre de 2019.) Disponible en: http://ppct.caicyt.gov.ar/index.php/rac/article/view/1472/pdf

3. Butany J, Nair V, Naseemuddin A, Nair GM, Catton C, Yau T. Cardiac tumours: diagnosis and management. Lancet Oncol. 2005;6:219-28.

4. Lima PP, López-Almodóvar LF, Jiménez JI, Orradre JL, Cañas A. Sarcoma cardiaco primario que comienza como dolor abdominal e isquemia intestinal: a propósito de un caso y revisión de la literatura. Cir Cardiovasc. 2013;20:156-8.

5. Neragi-Miandoab S, Kim J, Vlahakes GJ. Malignant tumours of the heart: a review of tumour type, diagnosis and therapy. Clin Oncol. 2007;19:748-56.

6. Burke A. Primary malignant cardiac tumors. Semin Diagn Pathol. 2008;25:39-46.

7. Chaves A, Alvarado Arce EM, Dam Arce C, Castillo Aldeano O, Induni López $\mathrm{E}$, Méndez Jiménez $\mathrm{E}$, et al. Sarcoma primario del pericardio, reporte de caso. Rev Clin Esc Med UCR-HSJD. 2018;8(1). Disponible en: https://revistas.ucr.ac.cr/index.php/clinica/article/view/32656

8. Perchinsky MJ, Lichtenstein SV, Tyers GF. Primary cardiac tumors: forty years' experience with 71 patients. Cancer. 1997;79:1809-15.

9. Li Z, Hsieh T, Salehi A. Recurrent cardiac intimal (spindle cell) sarcoma of the left atrium. J Cardiothorac Vasc Anesth. 2013;27:103-7.

10. Cho GJ, Kim HJ, Kang JS. Primary cardiac sarcoma in pregnancy: a case report. J Korean Med Sci. 2006;21:940.

11. Abad C. Tumores cardíacos (I). Generalidades. Tumores primitivos benignos. Rev Esp Cardiol. 1998;50:10-20.

12. Rottier R, Vega G. Tumor primario del corazón más común: mixoma cardíaco. Revista Médica de Costa Rica y Centroamérica. 2012;69: 481-7.

13. Modi A, Lipnevicius A, Moorjani N, Haw M. Prolonged survival with left atrial spindle cell sarcoma. Interact Cardiovasc Thorac Surg. 2009;8:703-4.

14. García EG, Dolores VB, Zaunbos MV. Sarcoma cardiaco primario: reporte de un caso y revisión de literatura actual. Reva Mex Cardiol. 2010;21:6.

15. Shanmugam G. Primary cardiac sarcoma. Eur J Cardiothorac Surg. 2006;29:925-32

16. Blackmon SH, Reardon MJ. Surgical treatment of primary cardiac sarcomas. Tex Heart Inst J. 2009;36:451-2.

17. Donsbeck AV, Ranchere D, Coindre JM, Le Gall F, Cordier JF, Loire R. Primary cardiac sarcomas: an immunohistochemical and grading study with long-term follow-up of 24 cases. Histopathology. 1999;34:295-304.

18. Blackmon SH, Patel A, Reardon MJ. Management of primary cardiac sarcomas. Expert Rev Cardiovasc Ther. 2008;6:1217-22. 